\title{
Late Onset Pompe Disease Mimicking Rigid Spine Syndrome
}

\author{
Francis B. Panosyan, Michael F. Fitzpatrick, Charles F. Bolton
}

Can J Neurol Sci. 2014; 41: 286-289

Pompe disease, also known as glycogen storage disease type II or lysosomal acid maltase deficiency, is an inherited disorder due to mutations that has an effect on the making of acid alphaglucosidase (GAA). Late onset Pompe disease (LOPD) is a subtype which is differentiated from the classic infantile form by partial deficiency of the affected enzyme GAA and milder symptoms. Hence, the onset of LOPD is delayed but can be as early as the first decade and as late as the sixth decade of life. ${ }^{1}$ It is a slowly progressive weakness of proximal muscles similar to limb girdle muscular dystrophy. Only one third have respiratory insufficiency, myalgia, cramps or symptoms and signs of myotonia, despite needle electromyography (EMG) recordings of myotonic discharges. Creatine phosphokinase (CPK) levels are frequently elevated.

Rigid spine syndrome (RSS) describes a slowly progressive muscular dystrophy which results in early contractures of the spinal extensor muscles with abnormal posture, severe scoliosis, marked respiratory muscle involvement and a relatively mild muscular weakness. The onset is generally in early childhood and it is associated with mutations in the selenoprotein N1 gene (SEPN1) on chromosome 1p36. Several differential diagnoses to RSS must be considered in a patient with prominent early spinal contractures; including Emery-Dreifuss muscular dystrophy (EDMD), Ullrich muscular dystrophy and Bethlem myopathy. However, early respiratory compromise is typically a useful feature that helps distinguish RSS from EDMD, Ullrich muscular dystrophy and Bethlem myopathy, where respiratory compromise is uncommon.

Here we report a case of late onset Pompe disease in a 71year-old male, who presented with symptoms and signs which initially suggested the rigid spine syndrome. Pompe disease is traditionally not included in the differential diagnosis of RSS; however, there is increasing evidence in the literature of similar cases, where late onset Pompe disease had phenotypic presentation of rigid spine syndrome..$^{2-4}$ Our patient's presentation was atypical for both Pompe disease and rigid spine syndrome. He had no proximal limb-girdle involvement despite the late presentation in the seventh decade of life and there was evidence of severe involvement of the diaphragm and thoracolumbar paraspinal muscles.

\section{Case Report}

A 71-year-old man was referred to the neuromuscular clinic after being seen initially in a movement disorder clinic for an unusual posture on standing. A neuromuscular problem was suggested. The patient reported that his postural changes started

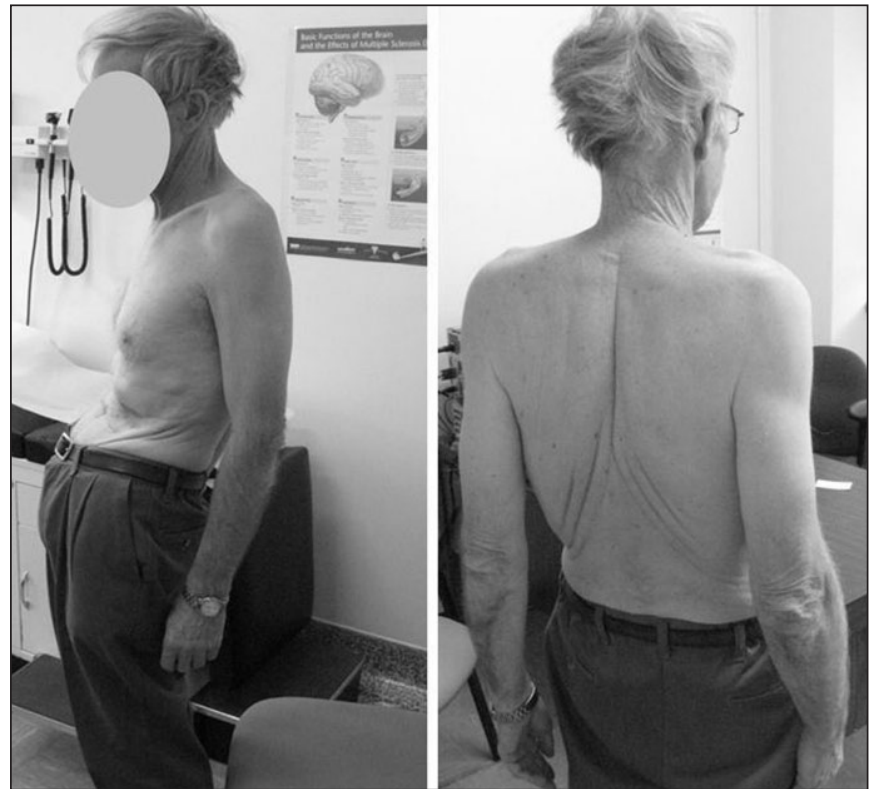

Figure 1: Involuntary peculiar slouched posture of the patient (chief complaint)

in childhood at the age of 14-15, but had significant deterioration ten years prior to his presentation. However, he felt that his posture seemed to have stabilized in the past several years. He found that in standing position, his abdominal muscle contracted pulling his pelvis forward. He maintained balance by leaning backwards with his arms thrown back (Figure 1). He had chronic aching in the lower thoracic and upper lumbar regions. He had a remote diagnosis of small fiber neuropathy affecting only the feet with mild distal sensory symptoms, but this did not really concern the patient. He reported no other significant neurological symptoms. He had no muscle stiffness or cramps. The patient denied any weakness in his extremities or dysphagia.

From the Division of Neurology (FBP, CFB) and Division of Respirology (MFF), Department of Internal Medicine, Queen's University, Kingston, Ontario, Canada

Received April 11, 2013. Final Revisions Submitted September 23, 2013. Correspondence to: Francis Panosyan, Connell 7, Division of Neurology, Kingston General Hospital, 76 Stuart St., Kingston, Ontario, K7L 2V7, Canada. Email: 4fbp@queensu.ca. 


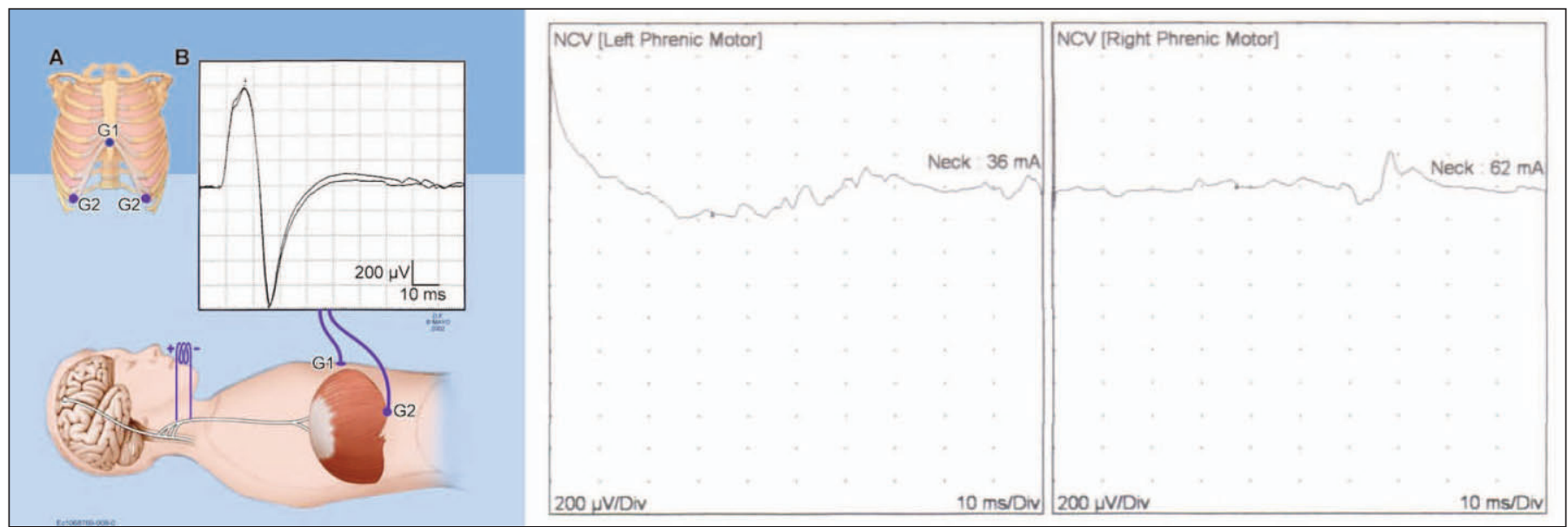

Figure 2: Nerve conduction studies of the phrenic nerves showing a typical normal response in comparison to absent response bilaterally from the patient.

He denied shortness of breath or orthopnea. The patient did not have any cognitive complaints, rash or diplopia. He was a retired radiation expert, but he reported minimal radiation exposure. His past medical history and family history were otherwise unremarkable.

On physical examination, he presented as a rather slim and intelligent man. His heart rate was regular and recorded at 66 beats per minute, blood pressure was $128 / 70$ and his oxygen saturation was $96 \%$ on room air. His mental status, language and cranial nerve examination were normal. No facial weakness or ptosis was noted. Muscle strength, bulk and tone were normal throughout his limbs. There was no evidence of myotonia. No fasciculations were noted in the tongue or limb muscles. He had no scapular winging. Deep tendon reflexes were somewhat reduced in the lower extremities but were all present. Plantar reflexes were flexor bilaterally. Sensory examination showed no abnormality to vibration, position or temperature. He had normal coordination in the limbs. He had a marked involuntary kyphosis of the spine with a thrusting forward of the pelvis. He had no limitation to flexion in his neck or spine. He was able to voluntarily correct this posture but only briefly. This posturing was also consistent as he walked. His posture relaxed in the supine position, but most interestingly he had marked thoracoabdominal paradoxical respiration with inward
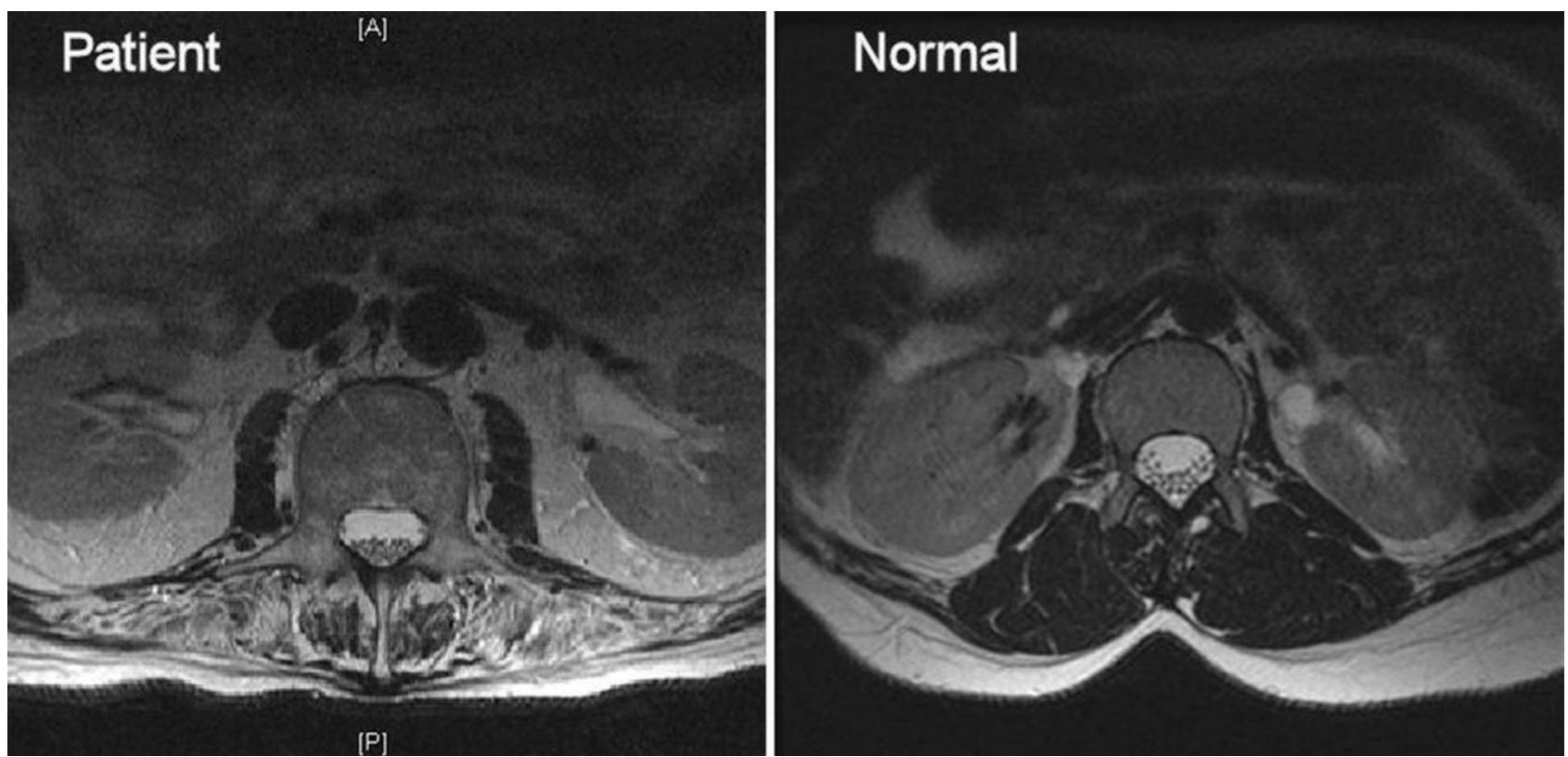

Figure 3: T2 axial MRI at the level of the kidneys showing severe atrophy with fatty replacement of the dorsal and lumbar musculature in our (patient) in comparison to a (normal) MRI from the archives at the same level. 
movement of his abdomen on inspiration in the supine position.

Nerve conduction studies (NCS) of the phrenic nerves bilaterally revealed absent responses. (Figure 2) Motor and sensory conduction studies of the R. Median nerve, including F waves were normal. Needle EMG of the diaphragm bilaterally showed absent motor unit potentials, no insertional activity and no spontaneous activity. Electromyogram of the thoracic and lumbar paraspinal muscles revealed decreased insertional activity, fibrillation potentials, myotonic and complex repetitive discharges in addition to myopathic motor unit potentials. Electromyographic studies of the upper limb, chest wall, cervical, iliopsoas and gastrocnemius muscles were all normal.

Investigations included normal complete blood count (CBC), electrolytes, creatinine, urea, erythrocyte sedimentation rate (ESR) and liver function tests. Mild elevation of creatine kinase 288 U/L (reference range: 55-197U/L) was noted. Normal thyroid stimulating hormone and vitamin $\mathrm{B}_{12}$ levels were found. A standing and supine chest radiograph was non diagnostic. Magnetic resonance imaging (MRI) of the spine revealed severe atrophy with fatty replacement of the dorsal and lumbar musculature. There was preservation of the psoas muscles and bilateral quadrates lumborum muscles (Figure 3). The patient was evaluated in the pulmonary function laboratory and it was found that his lung vital capacity was 2.72 litres and fell by $40 \%$ on changing from the erect seated position to the supine position. His respiratory rate increased to 24 breaths per minute in the supine position from 14 in the sitting position. Arterial blood gas analysis revealed moderate chronic respiratory acidosis with slightly reduced oxygen tension with $\mathrm{pH} 7.39 ; \mathrm{PCO}_{2}$ 53; $\mathrm{PO}_{2} 71$; $\mathrm{HCO}_{3} 31$ and $\mathrm{SO}_{2} 95 \%$.

A working diagnosis of RSS was made based upon the patient's bilateral diaphragmatic palsy, isolated atrophy of his lower thoracic and lumbar paraspinal muscles and sparing of his proximal limb muscles. He was followed in the respirology clinic and he was prescribed nocturnal bilevel positive airway pressure (BIPAP) to assist with his respiration in the supine position, however the patient could not tolerate it and stopped using BIPAP. He reported sleeping in the lateral position, with no sleeping difficulties or shortness of breath. He also continued to report restive sleep and no morning headaches despite declining the use of BIPAP.

Genetic testing was undertaken to test for SEPN1 mutations in the patient for rigid spine muscular dystrophy. SEPN1 gene testing showed no sequence variants that cause rigid spine muscular dystrophy. SEPN1 mutations are seen in only $40 \%$ of RSS. In considering his atypical presentation, late onset Pompe disease was contemplated as a differential diagnosis for his bilateral diaphragmatic palsy. As such, his serum was analysed for acid alpha-glucosidase activity. He remained stable during repeated outpatient clinical visits, with no further changes in his neurological or respiratory status. His alpha glucosidase enzyme activity testing showed marked reduction at 13 (reference range: 50-200). With clear evidence of reduced GAA activity, he was referred to genetic testing for GAA gene mutation and a working diagnosis of LOPD. The genetic testing result revealed that the patient had two mutations in the GAA gene. One mutation has a known association with a milder course of Pompe disease when present with another pathogenic variant. The other mutation has also been known to be associated with another pathogenic variant. These two mutations were consistent with a diagnosis of Pompe disease for our patient. He was provided with genetic counselling and informed that his children and grandchildren, who were all unaffected, could be tested if they wished as they might be carriers for this autosomal recessive disease. He was offered enzymatic replacement therapy (ERT) by the geneticists involved in his case, as this is currently covered by the Ontario Ministry of Health for patients with the diagnosis of Pompe. The patient declined enzyme replacement therapy at this time. He continues to be followed by a multi-disciplinary team to properly treat pulmonary, neuromuscular, orthopaedic and gastrointestinal elements of late onset Pompe disease.

\section{DisCuSSION}

Our patient presented with a main complaint of chronic difficulties with posture. Despite absence of a history of respiratory difficulties in terms of shortness of breath, orthopnea or headaches, bilateral diaphragmatic palsy was suspected during the clinical examination with the observation of marked thoracoabdominal paradoxical respiration. This case emphasizes the value of evaluating patients in supine position and watching for paradoxical movement of the diaphragm, especially with a suspicion of compensated symptoms. Needle EMG of the diaphragm was done using a published technique, where it would be difficult not to place the needle in the diaphragm. ${ }^{5}$ Once done, any absence of the insertional activity, spontaneous activity, neuromuscular correction or motor unit potentials would be consistent with the replacement of the diaphragm with a fibrous and fatty tissue. Atrophy of thoracic and lumbar paraspinal muscles with preservation of limb, chest wall, and cervical paraspinal muscles were also confirmed with EMG studies. The normal extremity studies were helpful in ruling out nondystrophic myotonia as a cause for the observed myotonic discharges and complex repetitive discharges which were observed in the paraspinal muscles during EMG studies. Magnetic resonance imaging showed severe isolated atrophy and fatty replacement of the dorsal and lumbar musculature in the lower thoracic and lumbar regions. Given his bilateral diaphragmatic paralysis our patient was dependent on accessory muscle use for breathing. In addition to the extreme involvement of his thoracolumbar paraspinal muscles, his peculiar posture might have aided in the use of his accessory respiratory muscles in standing position. We initially considered RSS because of the spinal curvature and severe muscle atrophy limited to the spine and diaphragm. Against this diagnosis was the fact the spine was not rigid throughout, especially in the cervical area. However, genetic testing for the most common mutation involved in the rigid spine muscular dystrophy; namely, SEPN1 gene mutation, did not reveal any sequence variants that are known to cause rigid spine muscular dystrophy. Although Pompe disease is traditionally not considered as a differential diagnosis for rigid spine muscular dystrophy, we considered late onset Pompe disease in the differential diagnosis given his significant respiratory involvement and despite the lack of progressive proximal weakness in a limb-girdle distribution, which is the typical presentation of LOPD. Serum enzyme activity analysis for acid alpha glucosidase in our patient showed marked reduction, which suggested a diagnosis of LOPD. Genetic analysis of the GAA gene confirmed the presence of two 
mutations consistent with the diagnosis of Pompe disease. Given that LOPD has a heterogeneous clinical presentation that can mimic other neuromuscular disorders; our case emphasizes the importance of considering late onset Pompe disease in all adults who have respiratory muscle weakness as part of their presentation. With advent of ERT with alpha-glucosidase as an approved therapy for the treatment of patients with the diagnosis of LOPD, our case highlights the need to identify LOPD patients with respiratory involvement even in the absence of limb-girdle muscle weakness. This can be easily achieved with an initial evaluation of serum GAA enzymatic activity and subsequently confirmed with genetic analysis. We found the patient's decision to decline enzymatic replacement therapy reasonable given his seemingly compensated state, potential adverse effects of the treatment and the modest potential benefits of treatment at his age.

\section{ACKNOWLEDGEMENTS}

The authors thank Dr. Stuart Reid and Dr. Robert Chen for their evaluations in movement disorder clinics, which suggested a possible neuromuscular cause, Ms. Nancy Walsh for undertaking the nerve conduction studies, Dr. Jennifer McKenzie for evaluating the patient for genetic counselling, Dr. Shannon Venance and Dr. Michel Melanson for their informal inputs with regards to this case. We are also grateful to the patient for his cooperation in this work. We previously published this case as an abstract in the 47th Annual Congress of the Canadian Neurological Sciences Federation, 2012.

\section{REFERENCES}

1. Hagemans ML, Winkel LP, Hop WC, Reuser AJ, Van Doorn PA, Van der Ploeg AT. Disease severity in children and adults with Pompe disease related to age and disease duration. Neurology. 2005;64(12):2139-41.

2. Fadic R, Waclawik AJ, Brooks BR, Lotz BP. The rigid spine syndrome due to acid maltase deficiency. Muscle Nerve. 1997; 20(3):364-6.

3. Kostera-Pruszczyk A, Opuchlik A, Lugowska A, et al. Juvenile onset acid maltase deficiency presenting as a rigid spine syndrome. Neuromuscul Disord. 2006;16(4):282-5.

4. Laforet P, Doppler V, Caillaud C, et al. Rigid spine syndrome revealing late-onset Pompe disease. Neuromuscul Disord. 2010; 20(2):128-30.

5. Bolton CF, Chen R, Wijdicks EFM, Zifko UA. Neurology of breathing: Butterworth-Heinemann Medical; 2004. 